\title{
Universiteit
}

Leiden

The Netherlands

\section{Platons Kratylos und die Theologia Platonica des Proklos}

Berg, R.M. van den; Perkams M., Piccione R.M.

\section{Citation}

Berg, R. M. van den. (2006). Platons Kratylos und die Theologia Platonica des Proklos. Proklos: Methode, Seelenlehre, Metaphysik. Akten Der Konferenz In Jena Am 18.-20. September 2003, 35-48. Retrieved from https://hdl.handle.net/1887/8292

Version: $\quad$ Not Applicable (or Unknown)

License: $\quad$ Leiden University Non-exclusive license

Downloaded from: https://hdl.handle.net/1887/8292

Note: To cite this publication please use the final published version (if applicable). 


\title{
PLATONS KRATYLOS UND DIE THEOLOGIA PLATONICA DES PROKLOS*
}

\author{
ROBERT M. VAN DEN BERG \\ "Sokrates scherzt im Kratylos und ist gleichzeitig ernsthaft" \\ Proklos, Theol. Plat. V 3, 16, 3-4
}

\section{Einleitung}

Platons Kratylos, sein Dialog über die Beziehung zwischen Sache und Name, zwischen Welt und Sprache, gehört zu den Favoriten der gegenwärtigen Platon-Forschung. Er ist eine nicht versiegende Quelle von Büchern und Artikeln, und das ist kaum erstaunlich, denn das Thema des Kratylos gehört zu den wesentlichen Fragen der Philosophie, die genaue Absicht, die Platon in dem Dialog verfolgt, ist jedoch rätselhaft. Dieses Interesse am platonischen Kratylos ist etwas Neues. Aus den Untersuchungen zur Rezeption des Kratylos im Altertum, die das Thema meines derzeitigen Forschungsprojektes ist, hat sich ergben, dass sich nur wenige antike Platoniker ausdrücklich mit dem Kratylos beschäftigten, obwohl viele - wie zahlreiche beiläufige Anmerkungen zeigen - ihn gelesen haben. Proklos stellt hier eine Ausnahme dar. Er ist der einzige Platoniker, von dem wir genau wissen, dass er einen Kommentar zum Kratylos geschrieben hat. Und dieser Kommentar ist glücklicherweise zu großen Teilen erhalten.

In meinem Beitrag möchte ich diesem bemerkenswerten Interesse des Proklos am platonischen Kratylos genauer nachgehen. Meiner Meinung nach besteht eine enge Beziehung zwischen dem KratylosKommentar und dem Hauptwerk des Proklos, der Theologia Platonica, in der er den anspruchsvollen Versuch unternimmt, aus den scheinbar unzusammenhängenden theologischen Bemerkungen in den Werken Platons eine systematische Theologie zu gewinnen. Um es ein wenig provokant zu formulieren: Proklos hat angefangen den Kratylos auf

\footnotetext{
* Ich danke Herrn Dr. Matthias Perkams herzlich für seine Hilfe beim Deutschen. Ein Stipendium der NWO (Netherlands Organisation for Scientific Research) hat die Abfassung dieses Beitrages ermöglicht.
} 
systematische Weise zu lesen und zu kommentieren, weil er glaubte, ein gutes Verständnis dieses Dialoges sei nützlich für sein Vorhaben, eine platonische Theologie zu schreiben. Ich möchte hier versuchen zu zeigen, dass Proklos im Kratylos einen wichtigen Baustein für das Gebäude der Theologia Platonica erkannte und was genau die Bedeutung des Kratylos in diesem Unternehmen ist.

\section{Der Kratylos: ein Dialog über göttliche Namen}

Ein erster Hinweis auf die Bedeutung des Kratylos finden wir im ersten Buch der Theologia Platonica, wo Proklos angibt, auf welche Weise jeder Dialog Platons zur Konstruktion einer platonischen Theologie benützt werden kann. Über den Kratylos schreibt er folgendes:

Bezüglich der charakteristischen Merkmale der Götter werden wir auch vielen heiligen Gedanken im Symposion nachjagen, vielen im Kratylos, und vielen im Phaidon. Denn in jedem von diesen werden die göttlichen Namen mehr oder weniger ausführlich erwähnt, auf Grund derer diejenigen, die sich in der Theologie geübt haben, die charakteristischen Merkmale von ihnen mittels Argumentation leicht fassen (Theol. Plat. I 5, 25, 18-23).

Proklos' Interesse am Kratylos bezieht sich also auf die so genannten göttlichen Namen und ihre Bedeutungen. Im Rahmen der Theologia Platonica, also einer Arbeit, die von den Göttern handelt, ist das nicht erstaunlich. Aber auch der Kratylos-Kommentar beschäftigt sich vor allem mit den Erklärungen der göttlichen Namen, die Sokrates im Kratylos gibt. Proklos glaubt sogar, dass das der бxo le Ziel des ganzen Dialoges, um das sich alles dreht. Er sagt dazu:

Dass im Kratylos der große Platon es sich zum Ziel ( $\sigma x о \pi \delta \varsigma)$ macht, nicht die ersten und mittleren sowie die letzten Welten der Götter zu besingen, sondern nur ihre Merkmale, wie sie von ihren Namen offenbart werden (In Cra. CLXVI 90, 24-27).

Es scheint mir in dieser Hinsicht nicht ohne Bedeutung zu sein, dass der Kommentar, der nur den ersten Teil des Kratylos behandelt, gerade da aufhört, wo im Kratylos die Deutung der göttlichen Namen zu Ende geht. Es ist klar, dass etwas verloren gegangen ist, aber es kann gut sein, dass das nur die letzten Blätter des Kommentars gewesen sind, und nicht ein bedeutender Teil, weil Proklos den Rest des Dialoges niemals kommentiert hatte. 
Der Nachdruck, den Proklos der Deutung der göttlichen Namen verleiht, ist darum bemerkenswert, weil sie in der modernen Forschung fast keine Rolle spielen. Meist wird angenommen, dass das zentrale Thema des Dialoges die Richtigkeit der Namen ist, das heißt, ob diese Richtigkeit auf Konvention beruht oder ob es eine Art natürliche Richtigkeit gibt. Auch Proklos bespricht in seinem Kommentar ausführlich diese Frage der Richtigkeit der Namen, um zu dem Ergebnis zu kommen, dass es eine Art natürliche Richtigkeit der Namen gibt, weil die Namen den Sachen ähnlich sein sollten. Die Namen sollten auf irgendeine Weise die Sache, die sie benennen, abbilden. Man könnte das wahrscheinlich am besten so verstehen, dass Proklos meinte, er müsse die Richtigkeit der göttlichen Namen gesichert haben, bevor er sie als Bausteine für seine Theologie benutzen könnte. Ich werde jetzt zuerst auf Grund von Theol. Plat. I 29 bestimmen, was diese göttlichen Namen für Proklos eigentlich sind und weshalb er glaubt, dass man von ihnen etwas über das Göttliche erfahren kann. Danach werden wir sehen, wie er diese Theorie in Theol. Plat. IV in die Praxis umsetzt.

\section{Proklos' Theorie der göttlichen Namen (Theol. Plat. I 29)}

Übrig ist, so meine ich, auch etwas über die göttlichen Namen zu sagen. Denn im Kratylos hält Sokrates es für passend, die Richtigkeit der Namen vor allem im Fall der Götter zu zeigen (Theol. Plat. I 29, 123, $18-21)$.

Proklos widmet das letzte Kapitel von Theol. Plat. I dem Thema der göttlichen Namen, ein deutlicher Hinweis auf das Interesse, das er im Rahmen seiner theologischen Arbeit an dieser Frage hat. Die Bemerkung, dass Platon sich damit im Kratylos beschäftigt, stimmt mit der Erklärung des бxотó ç des Dialoges im Kratylos-Kommentar überein, die ich eben zitiert habe. Der Hinweis, dass Sokrates "vor allem" im Fall der Götter die Richtigkeit der Namen zeigt, ist wahrscheinlich eine Anspielung auf Cra. 397 b-c, wo Sokrates bemerkt, dass die Namen von Menschen ihnen oft nicht angemessen sind. Darum soll die Richtigkeit der Namen im Fall der Götter untersucht werden, weil das Göttliche immer unveränderlich besteht und die Bildung der Namen in Bezug auf sie wohl am richtigsten sein müsse. ${ }^{1}$

1 Vgl. Procl., In Cra. X 4, 3-24. 
In Kapitel 29 weist Proklos zuerst darauf hin, dass laut Platon im Parmenides das Eine, Gott selbst, ohne Namen oder 'Logos' ist. Es lohnt sich, die Bemerkungen des Proklos zu dieser Stelle in seinem Parmenides-Kommentar nachzuschlagen, weil er dessen Text im Licht der Theorie der Richtigkeit der Namen aus dem Kratylos versteht:

Man muss dabei bedenken, dass das Wesen des Namens von etwas notwendig bedeutet, dass dieser sich mit dem Benannten deckt, durch Auflösung in einfachere Namen oder Zerlegung in seine Buchstaben. Wenn das so ist, dann kann man das ,Eine' nur in Buchstaben zerlegen, denn es ist nicht aus einfacheren Namen zusammengesetzt. Deshalb müssen die Buchstaben also etwas von seiner Natur vergegenwärtigen. Aber stellte jeder etwas anderes dar, dann wäre das Erste nicht eins. So dass das Eine, wenn es einen Namen hätte, nicht eins wäre. Dieses alles ist im Kratylos durch die Gesetze über die Namen ganz klar gezeigt“ (In Prm. VII 508, 89-509, 97 Steel; übers. von R. Bartholomai)

Wie gesagt, glaubt Proklos, die Richtigkeit der Namen bestehe darin, dass sie den Sachen ähnlich sind. Er spricht in diesem Zusammenhang sogar von "den Gesetzen über die Namen" (canonibus de nominibus). ${ }^{2}$ Wie wir hier erfahren, ist mit dieser Ähnlichkeit auch die Bedeutung des Namens gegeben. Ein Name ist einer Sache ähnlich/bedeutet diese Sache, entweder weil dieser Name in einfachere Namen aufzulösen ist - das heißt, weil das Wort selbst aus mehreren Wörtern besteht, die eine Beschreibung der Sache bilden - oder weil die Buchstaben selbst bedeutungsvoll sind. Im Kratylos werden beide Möglichkeiten diskutiert. Sokrates analysiert zum Beispiel den Namen Kronos als eine Zusammensetzung aus xó@os (rein, ungetrübt) und Noũc; er bezeichne also einen großen Verstand. Was die bedeutungstragenden Buchstaben betrifft, nimmt er an, dass der Buchstabe $r$ Bewegung zum Ausdruck bringt (Cra. 426 c 1 ff.; vgl. Procl., In Cra. LXXXV 40, 7-9). Im Fall des Einen ("Ev) ist es klar, dass dieser Name keine weiteren Wörter enthält. Wenn man jedoch seine Buchstaben analysiert, so gibt es drei Buchstaben (den Spiritus einbegriffen), die drei verschiedene Dinge abbilden. Der Name "Ev ist also kein passender Name für das Eine, weil das Eine eine absolute Einheit ist, die jede Vielheit ausschlieBt.

Die Götter, die nach dem Einen kommen, haben dagegen Namen. In Theol. Plat. I 29 erweckt Proklos den Anschein, dass alle Götter nach dem Einen Namen haben, aber aus In Cra. LXXI ergibt sich, dass das

2 Vgl. Pl., Cra. 388 d: Der Namensgeber ist ein Gesetzgeber. 
nicht der Fall ist. Ich möchte hier nicht ausführlich auf diese schwer zu verstehende Stelle im Kratylos-Kommentar eingehen. Um es kurz zu machen: Proklos meint, dass den Henaden, weil sie dem unbenennbaren Einen so ähnlich sind, selbst auch keine eigenen Namen zukommen, dass sie jedoch nach dem, was an ihnen jeweils Teil hat, benannt werden. Den ersten göttlichen Namen finden wir auf der niedrigsten Stufe der noetischen Götter, wo ein Licht von ihnen heraus auf die anderen Götter scheint. Dieses Licht heißt sehr zutreffend Phanes, nach einem wichtigen Gott der Orphik. Die Klasse der Götter, die darauf folgt, sind die noetischen und noerischen, Götter, und es sind gerade die Namen von dieser Klasse, die wir bald näher betrachten werden.

Kehren wir jetzt zur Theol. Plat. I 29 zurück, so finden wir, dass Proklos drei Stufen von göttlichen Namen unterscheidet. Zuerst gibt es die "allerersten und wahrsten und wirklich göttlichen der Namen, die man bei ihnen selbst (den Göttern) lokalisieren soll". Sie sind die geheimen göttlichen Namen, die von den Göttern selbst benützt werden. Zweitens gibt es die Namen, die Abbilder der ersten sind (124, 6: $\delta \mu o t \omega \mu \alpha \tau \alpha)$. Proklos schreibt ihnen einen dämonischen Status zu (124, 6: $\left.\tau \tilde{\eta} \varsigma \delta \alpha \iota \mu v^{i} \alpha \varsigma \mu o i \varrho \alpha \varsigma\right)$. Damit ist nicht gemeint, dass sie nur von Dämonen verwendet werden. 'Dämonisch' heißt bei Proklos alles, was zwischen Göttern und Menschen steht, auch zum Beispiel Engel und Heroen. Wenn Proklos sagt, dass diese Namen den ersten Namen ähnlich sind, dann ist damit gemeint, dass sie wie alle göttliche Namen auf allen Ebenen der Realität eine Art Abbildung der Götter sind. Deshalb sind die zwei Gruppen von Namen einander ähnlich. Aber weil Namen, wie wir oben gemäß in Prm. VII gesehen haben, ein Ausdruck von Kenntnis sind, und die Götter sich selbst am besten kennen, sind die ersten Namen besser als die dämonischen Namen.

An dritter Stelle gibt es die göttlichen Namen, die von Menschen gebildet worden sind. Proklos betont die Unvollkommenheit dieser göttlichen Namen, die wir benutzen. Wenn die dämonischen Namen schon Abbildungen der wahren göttlichen Namen sind, so sind diese Namen noch weiter von den originalen Namen entfernt, so wie im Staat Buch X ein gemaltes Bett eine Abbildung eines konkreten, hölzernen Bettes ist, das selbst wieder eine Abbildung der unstofflichen Idee 'Bett' ist. Der Name ist "eine letzte Erscheinung der Götter"

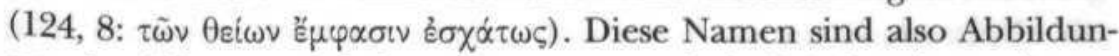
gen der Götter, nur keine sehr genauen, deshalb informieren sie uns über die Götter, aber es fehlt dieser Information an Exaktheit. Ihre 
Hersteller sind Experten im Bereich der Götter, die entweder von Göttern und Dämonen inspiriert sind oder ihren Intellekt benützen und auf diese Weise "bewegende Bilder ihrer inneren Visionen her-

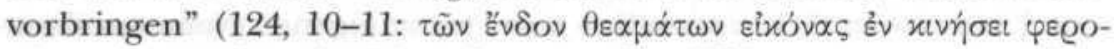

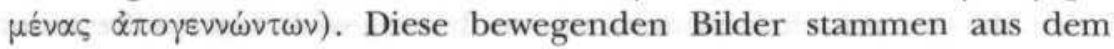
Phaidros-Mythos, der schon Theol. Plat. I 20 von Proklos erwähnt worden ist. Dem Mythos zufolge schauen die Seelen die zum Himmel empor steigenden "unwandelbaren Gesichte" (Phdr. 250 c 3: $\dot{\alpha} \varrho \varepsilon \mu \tilde{\eta}$ $\varphi \alpha \dot{\sigma} \mu \alpha \tau \alpha)$. Proklos setzt diese Erkenntnisse der gesegneten Seele den mangelhaften bewegenden Abbildungen entgegen, die die Götter

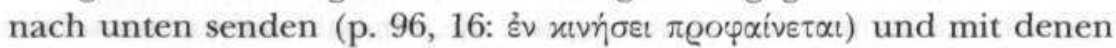
sich die meisten Menschen begnügen müssen.

Alles dreht sich also um die Tatsache, dass die göttlichen Namen uns, obwohl sie ein nützliches theologisches Instrument sind, nur einen sehr beschränkten Blick auf das Göttliche erlauben. Weil sie so weit vom Göttlichen entfernt sind, sind sie Bilder des Göttlichen auf

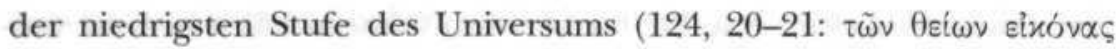
है $\sigma \chi \alpha \tau \omega \varsigma)$. In diesem Rahmen müssen wir den oft missverstandenen Vergleich von göttlichen Namen und theurgischen Statuen verstehen, den Proklos jetzt gibt:

Und wie Theurgie mittels bestimmter Symbole die generöse Gutheit der Götter hervorruft, um die künstlich hergestellten Statuen zu erleuchten, so zeigt auch die intellektuelle Erkenntnis des Göttlichen das verborgene Wesen der Götter mittels Zusammensetzungen und Trennungen (Theol. Plat. I 29, 124, 23-125, 2).

Theurgie ist bekanntlich die von gewissen Neuplatonikern geübte Magie, mit der man versuchte, die Götter herabzurufen, zum Beispiel mittels magischer Statuen, in die die Götter einziehen sollten. Nun gibt es bei Proklos so etwas wie Namenmagie, die Idee, dass gewisse göttliche Namen über magische Kräfte verfügen, mit denen man Götter herabrufen kann. Die Frage ist jetzt, welche Rolle diese Namenmagie in der Philosophie des Proklos spielt. Ich kann der Meinung nicht zustimmen, die zum Beispiel von Sarah Rappe in ihren Buch Reading Neoplatonism vertreten wird, ${ }^{3}$ dass die Theologia Platonica ein theurgischer Text ist und dass das Nennen von göttlichen Namen die Götter selbst herabruft, die dann den Leser der Theologia Platonica begeistern. Wenn man den oben zitierten Text genau betrachtet, sieht

3 S. Rappe, Reading Neoplatonism. Non-discursive Thinking in the Texts of Plotinus, Proclus, and Damascius, Cambridge 2000, 167-196. 
man, dass der Vergleichungspunkt ist, dass das unstoffliche Göttliche in etwas Stofflichem gezeigt wird (die 'Zusammensetzungen und Trennungen', von denen die Rede ist, sind die Laute der menschlichen Stimme). Mit Namenmagie hat das nichts zu tun, weil das verborgene Wesen der Götter, das die Namen zeigen, nur eine unvollkommene Abbildung der Götter ist, das Produkt der menschlichen Erkenntnis, und nicht, wie im Fall der theurgischen Statuen, die Götter selbst. In dem soeben erwähnten Text behauptet Proklos, dass die Leute, "die sich in der Theologie geübt haben" die charakteristischen Merkmale der Götter, die ihre Namen enthalten, "mittels Argumentati-

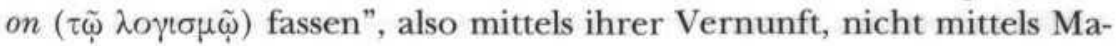
gie: Theologie handelt vom $\lambda$ ójọ über die Götter, nicht vom Wirken (हैo $\gamma \alpha$ ) der Götter, der Aufgabe der Theurgie.

\section{Die Theorie in der Praxis (Theol. Plat. IV)}

Nach der Darstellung von Proklos' Theorie der göttlichen Namen möchte ich nun erläutern, wie er diese Theorie in Buch IV der Theologia Platonica praktisch anwendet. Ich habe dieses Buch ausgewählt, weil es sich um die noetischen-und-noerischen Götter handelt (oi

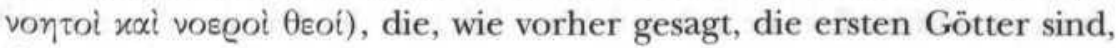
denen Namen beigelegt wurden, und weil die Namen hier eine wichtige Rolle in der Argumentation spielen. Die noetischen-undnoerischen Götter stehen zwischen den rein noetischen und den rein noerischen Göttern. Sie sind eine von Syrianos und Proklos auf Grund ihrer originellen Interpretation des Mythos aus Platons Phaidros eingeführte Novität in der Theologie des späten Neuplatonismus. Proklos ist sich durchaus bewusst, dass er seine Leser von der Richtigkeit seiner Interpretation eines so bekannten und oft kommentierten Textes überzeugen muss, und er greift dann auch die Konkurrenz scharf an, vor allem Theodoros von Asine. Sowohl Theodoros als auch Proklos berufen sich auf göttliche Namen; ihre Auseinandersetzung ist also für unseren Zweck sehr geeignet.

Doch wollen wir, bevor wir uns diesem Streit zuwenden, zuerst Kapitel 1 betrachten, wo Proklos diese Götter zum ersten Mal vorstellt. Weil die Götter bis jetzt unbekannt waren, muss er ihnen einen Namen verleihen. Proklos erklärt, dass diese Götter Gegenstände der Kontemplation von niedrigeren Götter sind - sie sind also 'noetisch' ( $($ oฑ $\tau$ Tó $)$ - aber auch selbst die rein noetischen Götter betrachten - 
sie sind also auch 'noerisch' (voegot). So geben es die Chaldaïschen Orakelsprüche, diese für die Neuplatoniker so wichtige Autorität, an:

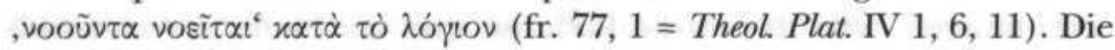
gemeinsame charakteristische Eigenschaft dieser Götter ist, dass sie auf diese Weise die noetischen und die noerischen Götter zusammenbringen. Um diese Eigenschaft deutlich zu machen, nennt Proklos sie also mit einem Namen, der die zwei Klassen von Göttern auch tatsächlich zusammenbringt. ${ }^{4}$ Bei näherer Betrachtung von Buch IV, die ich hier nicht unternehmen werde, würde sich herausstellen, dass Proklos jedes Mal, wenn er einen neuen Namen für eine Klasse von Götter einführt, erklärt, warum er glaubt, dieser sei ein zu diesen Göttern passender Name. Oft versucht er, den Namen mit der von den Göttern offenbarten Weisheit, entweder in den Chaldaisschen Orakelsprüchen oder anderswo, zu verbinden. Prokos hält also "die Gesetze über die Namen" (siehe In Prm. VII, wie oben S. 38) des Kratylos ein, die verordnen, dass die Namen eine Art Darstellung des göttlichen Wesens sein sollen.

Sehen wir uns jetzt Proklos' revolutionäre Interpretation des Phaidros-Mythos an. Der Mythos erzählt bekanntlich, wie die Olympischen Götter und die Seelen, die imstande sind, ihnen zu folgen, empor steigen zur "äußersten unterhimmlischen Wölbung". Wenn sie dorthin gelangt sind, stellen sie sich auf "den Rücken des Himmels", und weil sie von der kreisförmigen Bewegung des Himmels herumgeführt werden, schauen sie, was außerhalb des Himmels ist, "den überhimmlischen Ort", wo sie die Gerechtigkeit selbst, die Besonnenheit selbst, und so weiter sehen. Proklos glaubt, dass Platon in dem Mythos drei verschiedene Elemente unterscheidet:

1. die unterhimmlische Wölbung

2. den Rücken des Himmels, d.h. den Himmel selbst

3. den überhimmlischen Ort

Diese drei Elemente stellen laut Proklos drei Klassen von Göttern dar. Seine Meinung, dass auch der überhimmlische Ort eine Klasse von Göttern darstellt, lief vor allem der Interpretation des Theodoros von Asine zuwider. Dieser hatte in seiner Interpretation dem überhimmlischen Ort keine besondere Deutung zugeschrieben. Nach Meinung des Theodoros folgt der Himmel gleich dem Einen, weil die unter-

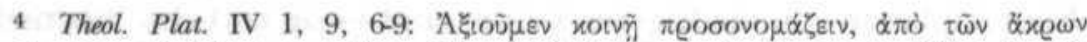

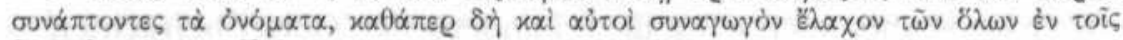

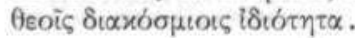


himmlische Wölbung der göttliche Intellekt (voũc) ist. Proklos sagt dazu folgendes:

Denn der Philosoph von Asine, von Plotin überzeugt, nennt das, was gleich dem Unsagbaren folgt, die unterhimmlische Wölbung, wie er in der Schrift Über die Namen über diese Sachen philosophisch aussagt (Theol. Plat. IV 23, 68, 15-17).

Proklos erwähnt die Argumentation des Theodoros In Ti. II, 274, 1823 und In Prm. VII 508, 80-85 Steel. Leider ist das genaue Argument schwer zu verstehen, aber Theodoros scheint sich auf die Form des Buchstabens E zu berufen, ${ }^{5}$ eine Technik der Analyse von Namen, die im Kratylos nicht erwähnt und später von Jamblich und Proklos verworfen wird, weil die Formen der Buchstaben auf reinem Zufall beruhen. ${ }^{6}$

Proklos versucht, Theodoros jetzt mit seinen eigenen Waffen zu schlagen und kommt auf Grund der göttlichen Namen zu folgendem Gegenargument:

Theodoros kann, weil er das Erste 'Himmel' (oð̉avóv) nennt, nicht akzeptieren, dass er (der Himmel) "der Blick, der sieht, was oben ist",

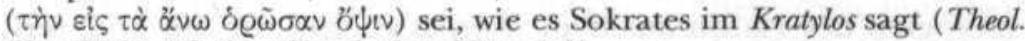
Plat. IV 23, 69, 16-17; vgl. Cra. 396 a 8 f.).

Was Proklos meint, ist, dass es dann, wenn man wie Theodoros den Himmel/den Uranos mit dem Ersten gleichsetzt, nichts gibt, was Uranos sehen könnte, wenn er, wie aus seinem Namen folgt, empor blickt. $\mathrm{Zu}$ diesem Argument bemerkt Proklos auch noch, dass die Interpretation des Theodoros die Beschreibung des überhimmlischen Ortes, wie er von einem begeisterten Sokrates im Phaidros besungen wird, nicht berücksichtigt.

W. Deuse hat in seiner Sammlung der Testimonien des Theodoros an dieser Stelle bemerkt, dass Theodoros die Etymologie des Kratylos ablehnt, und zieht hieraus die Schlussfolgerung, dass Theodoros sich offenbar keineswegs streng verpflichtet fühlt, die Aussagen Platons unangetastet zu lassen und seinen Systementwurf dem Texte Platons anzupassen. ${ }^{7}$ Meiner Meinung nach lehrt diese Stelle uns jedoch mehr über Proklos als über Theodoros. Theodoros hat die Etymologie des Kratylos nicht abgelehnt, er hat sie mit Recht nicht berücksichtigt. Denn es handelt sich hier um Proklos' Deutung der Kratylos-Stelle,

5 Siehe dazu H.D. Saffrey/L.G. Westerink, Proclus. Théologie Platonicienne IV, Paris 1981, xxv-xxvii.

6 Siehe Procl., In Ti. II, 278, 8-11.

7 W. Deuse, Theodore von Asine. Sammlung der Testimonien und Kommentar, Wiesbaden 1973, 98-101 (Kommentar zum Testimonium 8). 
der es aber an Überzeugungskraft fehlt. Betrachten wir zuerst die $\mathrm{Kra}$ tylos-Stelle selbst. Wie wir gesehen haben, ist mit Kronos laut Sokrates im Kratylos ein reiner (koros) Nus gemeint. Sokrates fährt wie folgt fort:

Dieser Kronos selbst ist wiederum ein Sohn des Uranos, und mit Recht wird die Hinaufsicht zur Höhe mit diesem Namen die himmli-

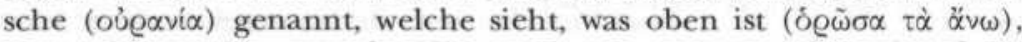
von woher ja eben, wie die Himmelskundigen sagen, der reine Geist hervorkommen soll, so dass also Uranos seinen Namen mit Recht führt (Cra. 396 b c; übers. v. F. Schleiermacher).

Sokrates meint also, dass ein himmlischer Blick, gerichtet auf das, was oben ist, den Himmel also, einen reinen Geist zustandebringt, nicht dass der Himmel selbst empor blickt. Es ist deshalb bemerkenswert, dass diese Deutung, die Proklos ausführlicher im Kratylos-Kommentar übernimmt (In Cra. CX, vor allem 60, 18-61, 7), später von Damaskios (Pr. II 91, 24-92, 4 Westerink/Combès) wiederholt wird. Diese Stelle ist kennzeichnend für die Weise, in der Proklos die göttlichen Namen in der Theologia Platonica benutzt. Er versucht nicht so sehr, selber die Namen zu etymologisieren, als vielmehr, die schon bestehenden Etymologien des Platon aus dem Kratylos ebenso wie aus anderen Dialogen in sein theologisches Gebäude einzufügen, was oft zu einer neuplatonischen Umdeutung der Etymologien führt.

\section{Der Kratylos: eine Theogonie}

Wie wir soeben gesehen haben, war Proklos nicht der einzige Neuplatoniker, der versuchte, auf Grund von göttlichen Namen zu einem besseren Verständnis der Götter zu gelangen. Andere, wie zum Beispiel Theodoros, hatten das auch getan. Es gibt jedoch keinen Hinweis darauf, dass Theodoros oder irgendein anderer Neuplatoniker vor Proklos ein großes Interesse am Kratylos hatten. Meiner Meinung nach erklärt sich Proklos' Interesse aus der die Athener Neuplatonische Schule kennzeichnenden Auffassung von platonischer Theologie. ${ }^{8}$ Wie schon bemerkt, versucht Proklos, aus den Werken Platons eine systematische Theologie herzustellen. Kritiker dieses Unternehmens haben ihm jedoch vorgeworfen, dass man bei Platon nirgendwo eine Theologie findet, obwohl er ab und zu von Göttern redet. Proklos versuche also, etwas in den Werken Platons zu finden, was

8 Eine der Besonderheiten des Proklos gegenüber Plotin, worauf C. Horn in seinem Beitrag hinweist, s. pp. $25 \mathrm{f}$. 
nicht da sei. Proklos antwortet darauf, dass sein Lehrer Syrianos auf überzeugende Weise gezeigt hat, wie die zweite Hypothese von Platons Parmenides eine systematische und erschöpfende Darstellung aller Klassen der Götter bietet. Während die Theogonien darlegen, welche Götter welche anderen Götter hervorbringen, lernen wir aus der zweite Hypothese, wie eine Gruppe von Ursachen neue Gruppen von Ursachen hervorbringt, und so weiter. Diese Klassen von göttlichen Ursachen bilden eine ursächliche Kette vergleichbar mit den Stammbäumen der Theogonien. Die zweite Hypothese ist also so etwas wie eine platonische Fassung der Theogonien des von Göttern begeisterten Orpheus und Hesiod, die uns auch auf systematische Weise die Götter präsentieren. Syrianos, so berichtet es uns Proklos, hatte gezeigt, dass im Parmenides

[...] jede der göttlichen Klassen von Plato auf symbolische Weise benannt worden ist und alle mittels philosophischer Namen zum Ausdruck gebracht werden, aber weder besungen mittels der Namen, die

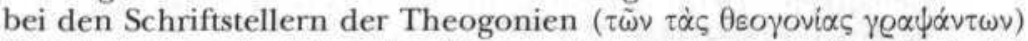

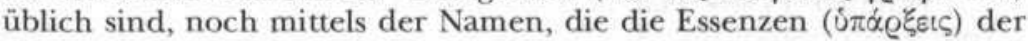
Götter bezeichnen, so wie die von den Göttern offenbarten göttlichen Eponyme der göttlichen Klassen es machen. ${ }^{9}$ Sondern, wie gesagt, werden sie mittels der Namen bezeichnet, die den Philosophen bekannt sind, wie 'das Ganze', 'die Menge', 'das Unbeschränkte', und 'die Grenze', Namen, die geeignet sind, um sie ihnen zu verleihen (In Prm. VI 1061, 35-1062, 7). ${ }^{10}$

Proklos stellt hier also die philosophischen Fachausdrücke des Parmenides den göttlichen Namen der Theogonien und denen, die mittels Orakel von den Göttern selbst offenbart worden sind, gegenüber. Gerade an diesem Punkt ist der Kratylos für das theologische Unternehmen des Proklos sehr nützlich. Proklos und seine Schule haben es sich nicht nur zur Aufgabe gemacht, eine platonische Theologie zu entwickeln, sondern auch, ihre Richtigkeit zu beweisen, indem sie ihre Übereinstimmung mit den von den Göttern offenbarten Theologien

9 Das heißt, alle Götter, die zur selben Kette (seirā) gehören, werden nach

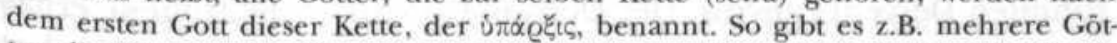
ter, die 'Dionysos' heißen, nach einem 'Ur-Dionysos'; s. Procl., In Cra. LXXXI 38, 5-21.

10 Siehe zu diesem Text und seiner Bedeutung C. Steel, 'Le Parménide est-il le fondement de la Théologie Platonicienne?', in: A.P. Segonds/C. Steel (Hgg.), Proclus et la Théologie Platonicienne. Actes du Colloque International de Louvain, 13-16 mai 1998, Leuven/Paris 2000, 373-397. S. zum Thema der Theogonien auch H.D. Saffrey, 'Accorder entre elles les traditions théologiques: une caractéristique du néoplatonisme athénien', in: E.P. Bos/P.A. Meijer (Hgg.), On Proclus and his Influence in Medieval Philosophy, Leiden 1992, 35-40; vgl. p. 45 zum Kratylos. 
und Theogonien zeigen. Im Kratylos übersetzt Platon die traditionellen Namen der Götter, wie sie aus Theogonien und Orakeln bekannt sind, in philosophische Sprache und ermöglicht es, platonische Theologie und Theogonie zu verbinden. Der Kratylos bildet also die Brücke zwischen Platon und den Theologen. Jemand wie Theodoros hatte niemals die Idee gehabt, orphische Theogonien neben die platonische Philosophie zu stellen. Für ihn war nur die Bedeutung von philosophischen Namen wie tò "Ev wichtig. Der Kratylos hat ihn also kaum interessiert.

Über die Relation zwischen Orpheus und Platon sagt Proklos in Buch IV der Theologia Platonica folgendes:

[...] Platon selbst im Kratylos, nach dem Vorbild der Orphischen Theogonien, nennt den Vater des Zeus Kronos, und den des Kronos Uranos. Der Wahrheit über sie durch ihre Namen nachspürend, erklärt er, dass der eine der Gestalter des Universums ist, der zweite der Behälter des göttlichen Intellektes, der dritte das Denken der ersten Gedanken. 'Denn der Blick, der sieht, was oben ist', sagt er 'ist der Himmel' (Theol. Plat. IV 5, 21, 15-21).

Diese Erklärung des Namens 'Uranos' durch das Denken der ersten Gedanken ist natürlich eine Anspielung auf Proklos' eigene Deutung der Etymologie dieses Namens, die wir soeben gesehen haben. Was die orphische Theogonie im Kratylos anbetrifft, bedauert Sokrates es, nachdem er die Namen des Uranos, Kronos und Zeus erklärt hat, dass er sich an 'die Genealogie des Hesiod' ( $\mathrm{Cra} .396 \mathrm{c}$ ) nicht weiter erinnern kann, während er in Cra. 402 b-c, mit Hinweis auf Hesiod, zwei Verse aus der Theogonie des Orpheus zitiert. Proklos, der die Genealogie des Hesiod mit Orpheus verbindet, betont selbstverständlich in seinem Kommentar die orphischen Elemente (siehe z. B. In Cra. CXV $66,28-67,23) .{ }^{11}$

Ich möchte diesen Beitrag abschließen mit der vielleicht etwas sonderbaren Bemerkung, dass Proklos die Absicht Platons zum Teil richtig verstanden hat. Für Platons philosophische Deutung einer Theogonie gibt es zeitgenössische Parallelen, zum Beispiel den berühmten Derveni-Papyrus. Der Derveni-Papyrus - die früheste erhaltene griechische Handschrift! - , der aus dem vierten Jahrhundert vor Christi Geburt stammt, enthält ein kosmogonisches Gedicht, das

$11 \mathrm{Zu}$ den orphischen Elementen in Procl., In Cra. s. auch L. Brisson, 'La figure du Kronos orphique chez Proclus. De l'orphisme au néoplatonisme, sur l'orgine de l'être humain', in: P. Bourgeaud (Hg.), L'orphisme et ses écritures. Nouvelles recherches (Revue de l'histoire des religions 219), Paris 2002, 435-458. 
dem Orpheus zugeschrieben wird, samt einem Kommentar. Laut dem anonymen Autor des Kommentars ist vieles aus der traditionellen Religion, Mythen ebenso wie Rituale, wenn es wörtlich genommen wird, sehr seltsam, so wie auch das kommentierte Gedicht. Deshalb muss man, wie er es macht, diese Sachen auf allegorische Weise interpretieren, um sie so mit den neuesten wissenschaftlichen Einsichten versöhnen zu können. Statt Wissenschaft darf man hier auch Philosophie lesen, was bekanntlich in dieser Zeit auf dasselbe hinausläuft. Ein beliebtes Instrument des Autors des Derveni-Papyrus ist die Etymologie. Viele moderne Gelehrte haben denn auch auf die Verwandtschaft zwischen dem Kratylos und dem Papyrus hingewiesen, und es gibt sogar den Vorschlag, dass derselbe Euthyphron, dem Sokrates seine etymologische Inspiration zuschreibt (Cra. 396 d), der Autor des Papyrus ist. ${ }^{12}$

Aber hatte Platon mit den Etymologien der göttlichen Namen im Kratylos eine Parodie auf Texte wie den Derveni Papyrus vorgehabt oder sollen wir sie doch in gewisser Weise ernst nehmen? Die moderne Forschung meint fast einstimmig, dass die Etymologien des Kratylos zum Ziel haben zu zeigen, dass die Etymologie kein geeignetes philosophisches Instrument ist, sondern nur zur Verirrung führen kann. Während Timothy Baxter meint, es handele sich hier um eine Parodie auf eine ganze Reihe griechischer Denker und Dichter, behauptet z. B. Rachel Barney, dass Platon uns diese ungeheuer lange Reihe von Etymologien nur deswegen präsentiert, um zu zeigen, dass er nicht unfähig ist, Etymologien zu bilden, obwohl er sie als ungeeignete Instrumente der Philosophie ablehnt. Er bildet sie sogar besser als jeder andere! Die Etymologien sind nach diesen Ansichten für Platon nur ein literarisches Spiel. ${ }^{13}$ Es gibt aber Ausnahmen, wie David Sedley, der in seinem neuen Buch zum Kratylos eine Lanze dafür gebrochen hat, die Etymologien ernster zu nehmen. Er weist zum Beispiel auf die Etymologien der Namen Kronos und Uranos hin, die wir schon erwähnt haben. Wenn Sokrates im Kratylos behauptet, dass mit Kronos ein reiner Intellekt (koros nous) gemeint ist, weil Kronos seinen Vater Uranos betrachtet, kann diese Etymologie von Platon doch nicht als völliger Unsinn gemeint sein. Platon regt bekanntlich im Timaios an

12 Siehe zu dieser Verwandschaft z. B. T.M.S. Baxter, The Cratylus. Plato's Critique of Naming, Leiden 1992, 130-139; zu Euthyphron s. C.H. Kahn, 'Was Euthyphro the Author of the Derveni Papyrus?' in: A. Laks/G.W. Most (Hgg.), Studies on the Derveni Papyrus, Oxford 1997, 55-63.

13 Baxter, The Cratylus, 86-106; R. Barney, Names and Nature in Plato's Cratylus, New York/London 2001, 69-73. 
einer berühmten Stelle zum Studium des Himmels (Uranos) an, weil dann, wenn wir die Regelmäßigkeit der Kreisläufe der Himmelskörper betrachten, unser Intellekt verbessert wird und wir auf diese Weise so weit wie möglich Gott ähnlich werden. ${ }^{14}$ So gibt es verschiedene Beispiele von Etymologien im Kratylos, die als Anspielungen auf spätere Dialoge verstanden werden können. Nicht alles ist selbstverständlich ernst gemeint, aber es ist auch nicht alles Spiel oder Parodie. Und obwohl man selbstverståndlich nicht mit der theologischen Deutung der Etymologien durch Proklos einverstanden sein kann, scheint es mir doch, dass er völlig Recht damit hat, dass Platon im Kratylos im Fall der göttlichen Namen versucht, eine Art Theogonie auf philosophische Weise umzudeuten, wie es z. B. auch der Autor des Derveni Papyrus tut. Es ist genau so, wie Proklos in Theol. Plat. V 3, 16, 3-4 sagt: "Sokrates scherzt im Kratylos und ist gleichzeitig ernsthaft".

14 D. Sedley, Plato's Cratylus, Cambridge 2000, 90-92. 